\title{
Age-dependent microstructural changes of the intervertebral disc: a validation of proteoglycan-sensitive spectral CT
}

\author{
Julian Pohlan ${ }^{1}$ (D) Carsten Stelbrink ${ }^{1} \cdot$ Matthias Pumberger ${ }^{2} \cdot$ Dominik Deppe $^{1} \cdot$ Friederike Schömig $^{2} \cdot$ Nils Hecht $^{3}$. \\ Friedemann Göhler ${ }^{1} \cdot$ Bernd Hamm ${ }^{1} \cdot$ Torsten Diekhoff $^{1}$
}

Received: 15 January 2021 / Revised: 23 March 2021 / Accepted: 29 April 2021 / Published online: 15 May 2021

(C) The Author(s) 2021

\begin{abstract}
Objective To analyze the two major components of the intervertebral disc (IVD) in an ex vivo phantom, as well as age-related changes in patients.

Methods Collagen and chondroitin sulfate were imaged at different concentrations in agar solution. Age-related changes in disc density were retrospectively analyzed in normal-appearing discs in dual-energy computed tomography (DECT) images from a patient cohort with various spinal pathologies $(n=136)$. All computed tomography $(C T)$ scans were acquired using single-source DECT at 80 and $135 \mathrm{kVp}$ with automatic exposure calculation. In 136 patients, the attenuation of normal-appearing discs on collagen/chondroitin maps (cMaps) correlated with the patients' age with Pearson's $r$ using standardized regions of interest in the anterior anulus fibrosus (AAF) and nucleus pulposus (NP).

Results DECT collagen mapping revealed concentration-dependent Hounsfield units (HU) of IVD components. For collagen, we found Pearson's $r=0.9610$ (95\% CI 0.6789-0.9959), $p=0.0023$ at $120 \mathrm{kVe}$, and $r=0.8824$ (95\% CI 0.2495-0.9871), $p=0.0199$ in cMap. For chondroitin sulfate, Pearson's $r$ was 0.9583 (95\% CI 0.6603-0.9956), $p=0.0026$ at $120 \mathrm{kVp}$, and $r=0.9646$ (95\% CI $0.7044-0.9963), p=0.0019$ in cMap. Analysis of normal-appearing IVDs revealed an inverse correlation of density with age in the AAF: Pearson's $r=-0.2294$ at $135 \mathrm{kVp}(95 \% \mathrm{CI}-0.4012$ to $-0.04203 ; p=0.0141)$ and $r=-0.09341$ in cMap $(95 \% \mathrm{CI}-0.2777$ to $0.09754 ; p=0.0003)$. In the NP, age and density did not correlate significantly at $135 \mathrm{kVp}(p=0.9228)$ and in $\mathrm{cMap}(p=0.3229)$. Conclusions DECT-based collagen mapping allows microstructural analysis of the two main intervertebral disc componentscollagen and chondroitin sulfate. IVD density declines with age, presumably due to a reduction in collagen and chondroitin sulfate content. Age-related alterations of disc microstructure appear most pronounced in the AAF.

Key Points

- DECT-based collagen mapping allows precise analysis of the two main intervertebral disc components—collagen and chondroitin sulfate.

- Intervertebral disc (IVD) density declines with age, presumably due to a reduction in collagen and chondroitin sulfate content.

- Age-related alterations of disc microstructure are most pronounced in the anterior anulus fibrosus (AAF).
\end{abstract}

Keywords Tomography, X-ray Computed $\cdot$ Spine $\cdot$ Collagen $\cdot$ Chondroitin sulfates $\cdot$ Intervertebral disc

\section{Abbreviations}

AAF Anterior anulus fibrosus

AEC Automatic exposure calculation

Julian Pohlan

julian.pohlan@charite.de

1 Department of Radiology, Campus Mitte, Charité -

Universitätsmedizin Berlin, Humboldt-Universität and Freie

Universität Berlin, Charitéplatz 1, 10117 Berlin, Germany
CI Confidence interval

cMap Collagen/chondroitin map

CT Computed tomography
2 Spine Surgery, Center for Musculoskeletal Surgery, Radiology, Charité - Universitätsmedizin Berlin, Campus Mitte, Humboldt-Universität zu Berlin, Freie Universität Berlin, Berlin, Germany

3 Department of Neurosurgery and Center for Stroke Research Berlin (CSB), Radiology, Charité - Universitätsmedizin Berlin, Campus Mitte, Humboldt-Universität zu Berlin, Freie Universität Berlin, Berlin, Germany 
DDD Degenerative disc disease

DECT Dual-energy computed tomography

HU Hounsfield unit

IVD Intervertebral disc

MRI Magnetic resonance imaging

NP Nucleus pulposus

ROI Region of interest

\section{Introduction}

Intervertebral disc (IVD) degeneration commonly occurs in the aging spine, often leading to degenerative disc disease (DDD) with low back pain $[1,2]$. The intervertebral disc connects two vertebral bodies and consists of cartilaginous and fibrous elements - the central nucleus pulposus (NP) and the surrounding anulus fibrosus [3]. Collagen and proteoglycans such as chondroitin are the main biochemical components of the disc and are thought to play a significant role in the development of DDD $[4,5]$.

Microstructural alteration of IVDs may occur before obvious morphological change becomes apparent and is attributed to reorganization of the extracellular matrix [6]. Importantly, imaging features of degeneration such as disc bulging, disc protrusion, and annular fissure also occur in asymptomatic individuals and increasingly with advanced age [7]. Magnetic resonance imaging (MRI) is the current reference standard for assessment of spinal conditions involving soft tissue with excellent morphological depiction of the IVD and early degenerative changes [8]. MRI provides additional information when sequences such as glycosaminoglycan chemical exchange saturation transfer (gagCEST) are used [9]. However, several contraindications limit the use of MRI in patients, with an ongoing debate about cardiac implants $[10,11]$.

Another diagnostic modality to assess for discogenic pain would be provocative discography, with contrast application after placing a needle in the IVD $[12,13]$. This procedure has been proven to be a safe and accurate diagnostic procedure [14]. Imaging findings of DDD are not only prevalent in symptomatic individuals but also highly prevalent among asymptomatic individuals [7]. Therefore, provocative discography, performed according to the International Association for the Study of Pain (IASP) criteria, remains the most specific procedure to diagnose discogenic low back pain $[12,15]$.

Dual-energy computed tomography (DECT) may facilitate depiction of the characteristic properties of collagen with relatively high attenuation of the densely packed fibers and a relatively low effective atomic number, thus improving visualization of tendons, ligaments, and other collagen-rich structures [16]. Recent studies suggest that DECT is also useful in depicting the morphology and microstructural changes of IVDs [17, 18]. Additionally, virtual non-calcium (VnCa) maps have been shown to increase diagnostic performance and confidence for depicting lumbar disc herniation as compared with standard CT [19]. However, a systematic analysis of quantitative and qualitative aspects has yet to be conducted.

Therefore, the aim of this study was to analyze major components of the IVD in a phantom setup using DECT and to determine whether the results may be transferred to age-related changes of IVD microstructure in patients.

\section{Material and methods}

\section{Ethics approval and informed consent}

This study was approved by the local ethics board under EA1/ 230/19. Patient consent was waived given the retrospective nature of the analysis.

\section{Phantom setup}

Collagen (Merck) and chondroitin sulfate (Merck) were separately added at five different concentrations to small vials, each containing $2 \mathrm{ml}$ of heated $2 \%$ agar solution, i.e., control agar (0)-67.8 mg collagen (1)-125.7 mg collagen (2)$252.0 \mathrm{mg}$ collagen (3) $-495.0 \mathrm{mg}$ collagen (4)-1005.0 mg collagen (5) and control agar (0)-67.4 mg chondroitin sulfate (1)-125.3 mg chondroitin sulfate (2)-251.0 mg chondroitin sulfate (3)-502.0 mg chondroitin sulfate (4)-1011.0 mg (5). All vials were thoroughly mixed. The phantom was surrounded by water during imaging.

\section{Patient recruitment}

We retrospectively included patients who underwent a DECT scan of the lumbar spine from Nov. 2014 to Feb. 2020. Patients without a recent MRI scan of the lumbar spine at least two months prior to or after the DECT scan were excluded. For analysis, one normalappearing IVD per patient was identified based on morphological appearance in both MRI and DECT datasets. Disc abnormalities such as bulging, disc segment adjacent to vertebral fracture, discitis, spondylitis, or severe artifacts (e.g., due to metal implants) resulted in the exclusion of the corresponding intervertebral disc. If eligible, L3/4 was chosen for further measurement. Otherwise, we selected $\mathrm{L} 2 / 3$ or $\mathrm{L} 4 / 5$ and so on for analysis, prioritizing a level close to L3/4 (Fig. 1). Only one disc was included per patient. If no normal lumbar disc was present, the scan was excluded from analysis. 


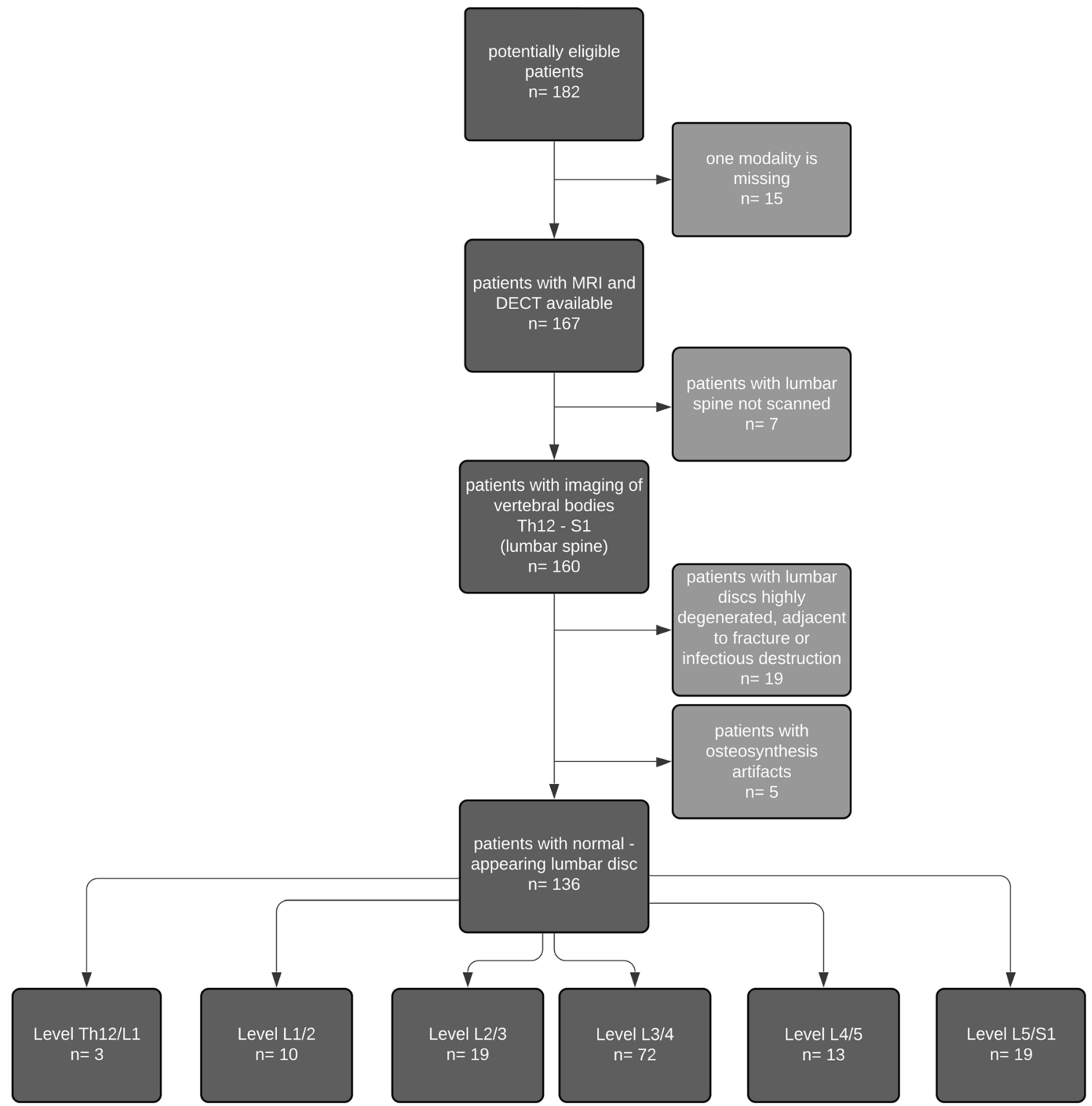

Fig. 1 Flow chart of patient selection. We retrospectively included patients from four separate studies who underwent CT and MRI for different indications. Specifically, we included patients who underwent CT-guided infiltration $(n=14)$, patients with axial spondyloarthritis $(n=$

\section{DECT scanning}

CT images were acquired in a conventional single-source CT scanner (Aquilion ONE Vision, Canon Medical Systems) equipped with a 320-row detector to allow repeated scanning without table movement. Dual-energy datasets were obtained using the technique of sequential acquisition of two volumes (135 $\mathrm{kVp}$ and $80 \mathrm{kVp}$ ). The phantom experiment was performed with ascending tube current $(10,20,30,40,50,70$, $90,110,130,150 \mathrm{~mA})$. Gantry rotation time was $0.275 \mathrm{~s}$ with a changeover time of $0.5 \mathrm{~s}$, resulting in an overall scanning time of $1.05 \mathrm{~s}$ per scan. Patients were imaged in a prone or supine feet-first position. Images were acquired using
75), patients with osteoporotic vertebral fractures $(n=68)$, and patients with suspected septic spondylodiscitis $(n=25)$, totaling $n=182$. All reasons for exclusion are provided

automatic exposure calculation (AEC) with a standard deviation of 12 Hounsfield units (HU), and the wide-volume technique was applied if the scan range exceeded $16 \mathrm{~cm}$.

\section{Postprocessing}

Dual-energy datasets were reconstructed with $0.5 \mathrm{~mm}$ slice thickness using a medium soft tissue kernel without beam hardening compensation and image-based iterative reconstruction (AIDR-3D, standard level). CT datasets were then further postprocessed at the CT console to generate collagen/ chondroitin maps (cMaps) using a collagen-specific gradient of 1.1 and applying a three-material-decomposition algorithm. 


\section{Ex vivo phantom measurement}

Images were transferred to a workstation with a highresolution monitor. Using Horos (Version 4.0, Horos project, based on OsiriXTM open source), we defined circular regions of interest (ROIs); all analyses were performed with $3 \mathrm{~mm}$ averaged slice thickness in axial reformation.

For the phantom setup, $371 \mathrm{~mm}^{2}$ standardized ROIs with a diameter of $21.7 \mathrm{~mm}$ were placed in representative areas of the vial containing collagen $(n=196)$ or chondroitin sulfate $(n=$ 175 ) at different concentrations. Three hundred eighteen ROIs were analyzed for the phantom experiment (excluding 53 ROIs for internal control, e.g., $\mathrm{H}_{2} \mathrm{O}$ ).

\section{Patient measurement}

A total of 136 IVDs from 136 patients were included in the analysis. One $30-\mathrm{mm}^{2}$ standardized ROI with a diameter of $6.2 \mathrm{~mm}$ each was placed in the NP and in the AAF, in oblique axial reformations of $3 \mathrm{~mm}$ slice thickness both in $135-\mathrm{kVp}$ images and in cMaps. A total of 544 ROIs, four in each patient, were analyzed for age correlation. Measured HU were exported using the "Export ROI" plugin and converted to an Excel file (Microsoft, Office 365 MSO).

Similarly, MRI standardized ROIs $\left(10 \mathrm{~mm}^{2}\right.$ for ROIs in the AAF and $20 \mathrm{~mm}^{2}$ for ROIs in the NP) were placed at sagittal short-tau intensity recovery (STIR) or turbo-inversion recovery-magnitude (TIRM) sequences at $4 \mathrm{~mm}$, and exported for further analysis. Intensity values were normalized by subtraction of intensities from $20 \mathrm{~mm}^{2}$ ROIs placed in the paravertebral musculature.

\section{Statistics}

Descriptive statistics were computed for the phantom and patient measurements. We performed Pearson's correlation analysis of the disc density data, as criteria for linearity were fulfilled. The paired $t$ test was used to compare densities measured in male and female patients. Age groups for density measurements were set to $<29,30-39,40-49,50-59,60$ $69,70-79,>80$. Analyses were performed using ANOVA. All statistics were conducted with IBM SPSS (Version 25.0) and GraphPad Prism (Version 8).

\section{Results}

\section{Phantom measurement}

Analysis of different concentrations of collagen solution yielded a high correlation of density and mass at $120 \mathrm{kVe}$ with $r=$ short-tau intensity recovery 0.9610 (95\% CI 0.6789 to 0.9959 ), $p=0.0023$ (Fig. 2). For cMaps, we found a correlation with $r=0.8824$ (95\% CI 0.2495 to 0.9871$), p=$ 0.0199 . The density gradient $(135 / 80 \mathrm{kV})$ was 1.01 for collagen and 0.8 for chondroitin sulfate.

Chondroitin sulfate displayed a significant concentrationdependent density in conventional CT scans with a positive linear correlation at $120 \mathrm{kVe}$ with Pearson's $r=0.9583$ (95\% CI 0.6603 to 0.9956 ), $p=0.0026$, and $r=0.9646$ (95\% CI 0.7044 to 0.9963$), p=0.0019$, in cMaps, respectively.

\section{Patient measurement}

Among the 182 patients who underwent DECT, MRI identified 136 patients with morphologically normal lumbar discs (see Fig. 1). The mean age of the patients included was 54.91 years (SD 18.90). The proportion of female patients was at $54.4 \%$. CT scans were performed with a mean dose-length product (DLP) of $476.83 \pm 366.17 \mathrm{mGy} * \mathrm{~cm}$ and with a computed tomography dose index volume $\left(\mathrm{CTDI}_{\mathrm{vol}}\right)$ of $13.95 \pm$ $8.85 \mathrm{mGy}$.

The NP showed a density of $84.6 \mathrm{HU} \pm 11.8$ at $135 \mathrm{kVp}$ and $98.0 \mathrm{HU} \pm 34.1$ in cMaps, the AAF $90.3 \mathrm{HU} \pm 11.0$ at 135 $\mathrm{kVp}$ and 107.1 HU \pm 34.1 in cMaps. Differences in density of the AAF compared to the NP were significant both at $135 \mathrm{kVp}$ (paired $t$ test; $p=<0.0001)$ and in cMaps $(p=0.0004)$.

One-way ANOVA identified significantly different disc density of the levels Th12/L1 and L5/S1 for both AAF and NP compared with the levels in between. Upon exclusion of Th12/L1 and L5/S1, there was no significant difference between IVD levels L1-L5 with regard to density at $135 \mathrm{kVp}$ and in cMaps (Table 1).

\section{Correlation with age}

There were morphological changes in aging subjects (Fig. 3). Measurement at $135 \mathrm{kVp}$ revealed a nonsignificant negative linear correlation of HU and age with Pearson's $r=-0.044$ (CI -0.211 to 0.125$)$ and $p=0.609$ for the NP. For the AAF, there was a weak negative linear correlation with $r=-0.217$ $(\mathrm{CI}-0.371$ to -0.050$)$ and $p=0.011$. Analysis of cMap densities of the NP yielded a significant negative linear correlation of HU and age with Person's $r=-0.201$ (CI -0.357 to $-0.034)$ with $p=0.019$. In cMaps, correlation for the AAF was $r=-0.418(\mathrm{CI}-0.548$ to -0.269$)$ with $p=<0.0001$ (Fig. 4).

Linear logistic regression of the ROI measurements in the $\mathrm{AAF}$ in correlation with age yielded a slope of $-0.14(95 \% \mathrm{CI}$ -0.25 to $-0.032 ; p=0.011)$ at $135 \mathrm{kVp}$ and $-0.77(95 \% \mathrm{CI}-$ 1.06 to $-0.488 ; p=<0.0001)$ in cMaps. The NP had a nonsignificant slope of -0.027 (95\% CI -0.13 to $0.077 ; p$ $=0.61)$ at $135 \mathrm{kVp}$ and $-0.37(95 \% \mathrm{CI}-0.68$ to $-0.063 ; p=$ 0.019 ) in cMaps. cMap density in the AAF was analyzed according to the respective age group, with significant differences in the ANOVA with $p<0.0001$. Density of the NP in 
Fig. 2 Results of density measurements in the phantom. Attenuation in $\mathrm{HU}$ for collagen and chondroitin sulfate at different concentrations measured in $120 \mathrm{kVe}$-equivalent and cMap reconstructions. Attenuation shows a steady increase with the concentration, which is more pronounced for chondroitin sulfate compared with collagen. $c$ Map $=$ collagen $/$ chondroitin map; $H U=$ Hounsfield unit
Collagen
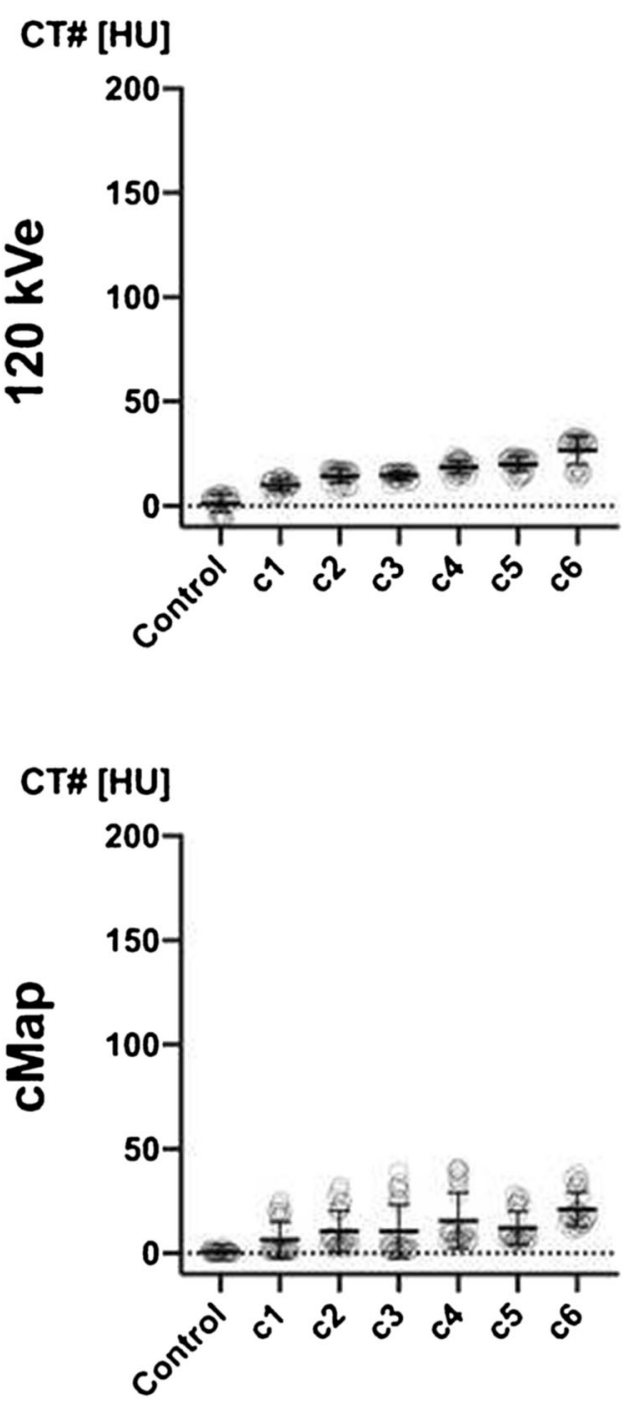

Chondroitin

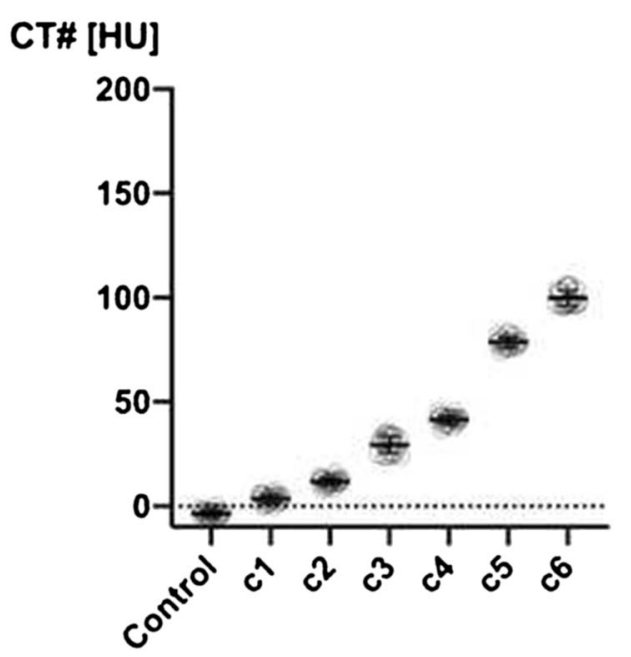

CT\# [HU]

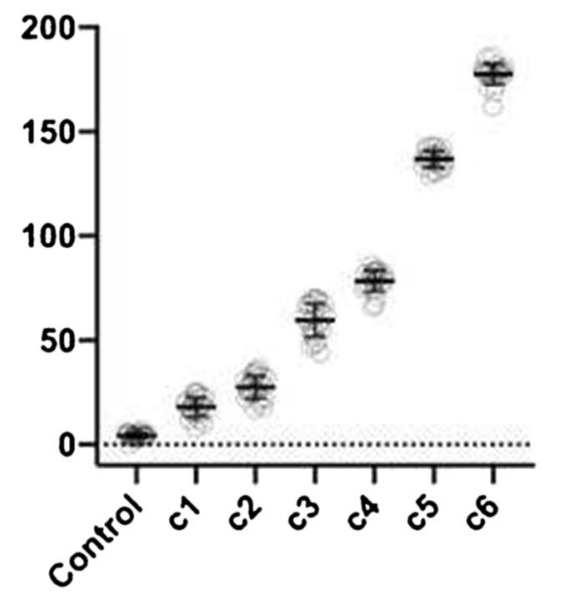

Table 1 Level-dependent density in human intervertebral discs

\begin{tabular}{lllll}
\hline Levels included & Th12-S1 & \multicolumn{3}{c}{ L1-L5 } \\
\hline Number of patients & $n=136$ & & $n=114$ & \\
Localization & $\mathrm{NP}$ & $\mathrm{AAF}$ & $\mathrm{NP}$ & $\mathrm{AAF}$ \\
$135 \mathrm{kVp}$ & $p=0.0494$ & $p=0.2355$ & $p=0.2724$ & $p=0.5937$ \\
cMap & $p<0.0001$ & $p=0.0046$ & $p=0.9937$ & $p=0.4805$ \\
\hline
\end{tabular}

As shown in the left side of the table, statistical analysis with one-way ANOVA identified significantly different disc density of the Th12/L1 and L5/S1 levels for both nucleus pulposus and anterior anulus fibrosus compared with the levels in between. Upon exclusion of Th12/L1 and L5/S1, shown in the right side of the table, there was no significant difference between intervertebral disc levels L1-L5 with regard to density at 135 $\mathrm{kVp}$ and in cMaps

$N P$ nucleus pulposus, $A A F$ anterior anulus fibrosus different age groups yielded no significant differences ( $p$ value $=0.30)$.

Gender differences in density analyzed between male and female discs were nonsignificant. At $135 \mathrm{kVp}$, the male AAF displayed a mean density of 91.1 HU $\pm 13.5 \mathrm{HU}$ compared with the female AAF density of $90.2 \mathrm{HU} \pm 11.1$ with $p=0.85$, whereas the male NP had 84.4 HU \pm 12.9 and the female NP 85.4 HU \pm 10.2 with $p=0.56$. In the cMaps, the male AAF density was $112.7 \mathrm{HU} \pm 33.9$ and female AAF 102.6 $\mathrm{HU} \pm$ 35.4 with $p=0.099$, while the male NP had a mean density of 102.0 HU \pm 35.2 and the female NP a mean of $96.4 \mathrm{HU} \pm 34.9$ with $p=0.379$.

STIR intensity values significantly correlated with age at the AAF with $p=<0.0001, r=-0.60(95 \% \mathrm{CI}-0.70$ to $0.48)$, and at the NP with $p=<0.0001, r=-0.74(95 \% \mathrm{CI}-$ 0.81 to -0.66 ) (see Fig. 4). 
Fig. 3 Morphology of intervertebral discs in different age groups. Age-dependent morphology of intervertebral discs in three patients of different ages: first column, aged 22: density of NP, $89.5 \mathrm{HU}$, and AAF, 100.5 $\mathrm{HU}$ at $135 \mathrm{kVp}$; NP, $113.8 \mathrm{HU}$, and AAF, $132.1 \mathrm{HU}$ in cMap; second column, aged 55: density of NP, 77.1 HU, and AAF, 101.4 $\mathrm{HU}$ at $135 \mathrm{kVp}$; NP, 99.6 HU, and AAF, 107.0 HU in cMap; and third column, aged 90 years: density of NP, $66.9 \mathrm{HU}$, and $\mathrm{AAF}, 76.6 \mathrm{HU}$ at $135 \mathrm{kVp}$; NP, $56.7 \mathrm{HU}$, and AAF, 85.0 HU in cMap. Images show sagittal DECT cMaps with corresponding sagittal CT scans; below, T1weighted MR images and T2weighted STIR images are shown. $D E C T=$ dual-energy computed tomography; MRI= magnetic resonance imaging

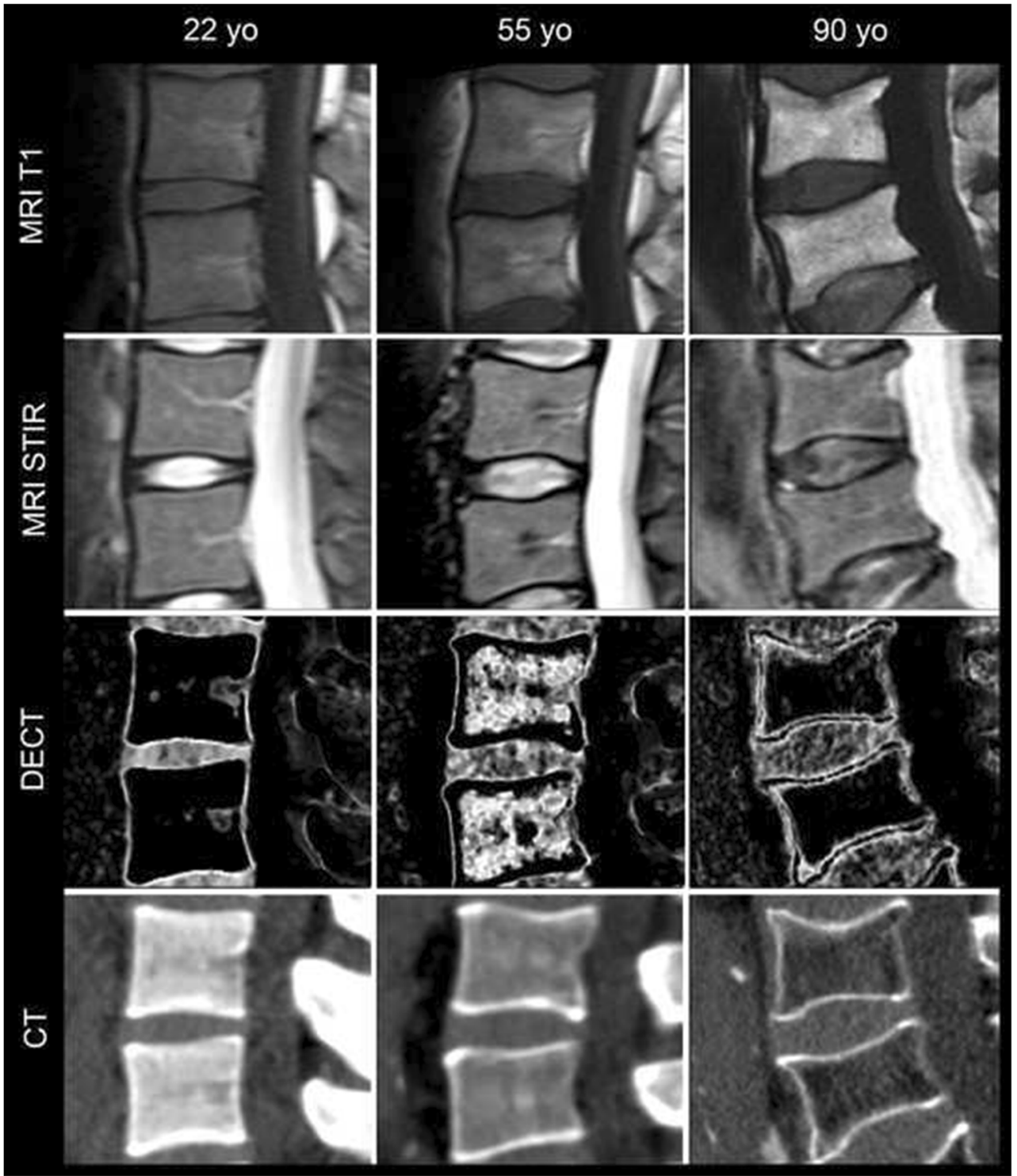

Correlation of MRI STIR intensity values with cMap densities yielded a significant correlation for the AAF with $p=$ $0.0002, r=0.32$ (95\% CI 0.16 to 0.46 ), and was nonsignificant for the NP with $p=0.41, r=0.07$ (95\% CI -0.098 to 0.24) (see supplementary Figure S1).

\section{Discussion}

This is the first study aimed at analyzing intervertebral disc components in a phantom setup using DECT and transferring the experimental data to age-related changes in IVD microstructure in patients.

Our phantom experiments show a concentration-dependent density of the discal components collagen and chondroitin sulfate on DECT imaging. However, chondroitin sulfate contributes more strongly to the densities measured in both conventional CT images and cMaps. Patient-based analysis indicates a steady age-dependent decline of density in the AAF and NP, which is better depicted by cMaps compared to conventional CT images. Age-dependent density loss appears to be more pronounced in the AAF than in the NP.

Our results suggest that DECT is capable of assessing the proteoglycan content of the IVD and might therefore be sensitive to disc degeneration and other conditions that affect microstructure and proteoglycan content. However, aging is a significant factor that requires consideration in assessing disc pathologies on DECT images. These results reflect the physiological loss of major disc components in normalappearing IVDs, which needs to be separated from pathological degeneration and disc destruction.

The age-dependent loss of both collagen and chondroitin sulfate we observed in the AAF and NP is strongly supported by a recent biochemical analysis of human IVDs [20]. 


\section{Nucleus}
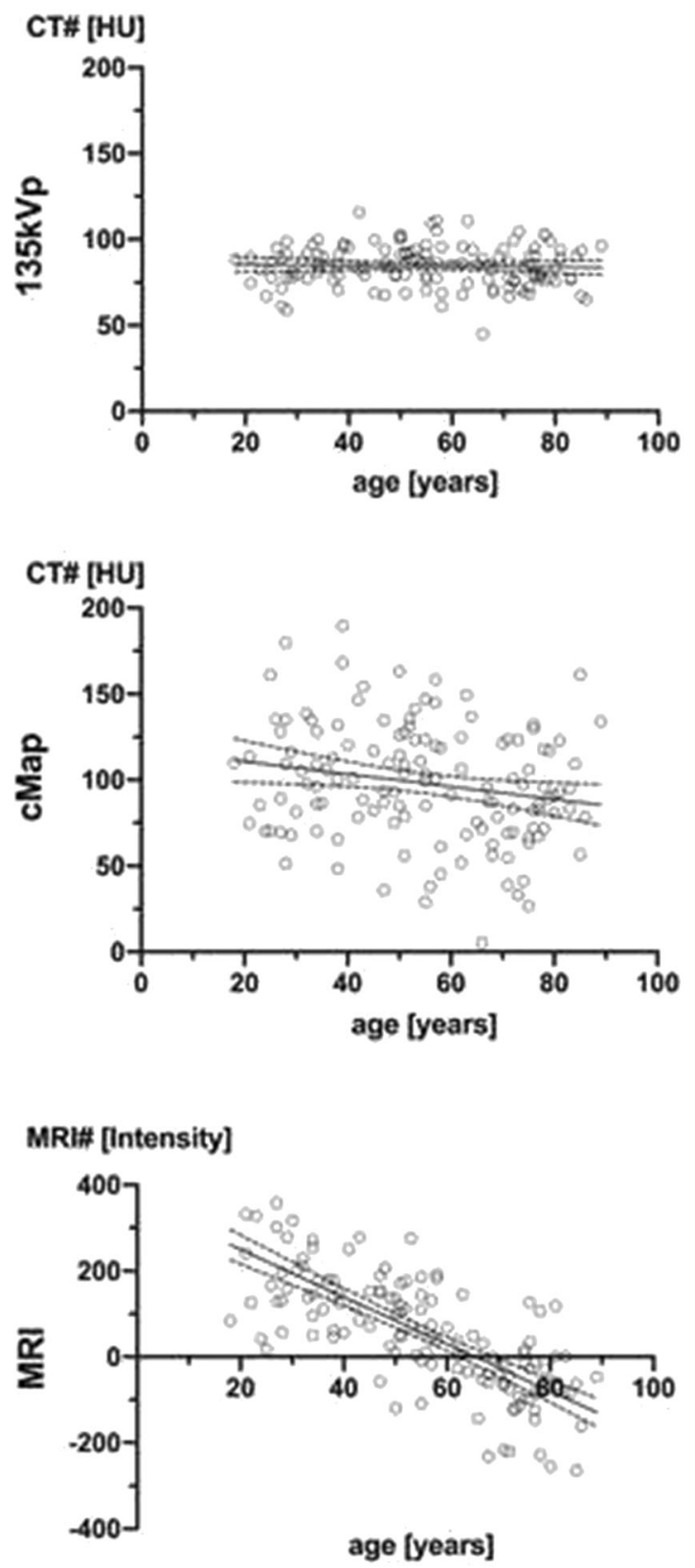

Fig. 4 Age-dependent cMap density and MR intensity of human intervertebral discs. Density of normal-appearing intervertebral disc (IVD) compartments measured in conventional $135-\mathrm{kVp}$ images and cMaps in correlation with patient age. There is no significant correlation in 135-kVp images for the NP $(p=0.65)$, whereas cMaps show significant correlation $(p=0.02$; Pearson's $r=-0.2)$. Conversely, $135-\mathrm{kVp}$ images show a significant correlation of HU with age in the AAF ( $p=$ $0.002 ; r=-0.27)$ and an even stronger correlation in the cMaps $(p<$

Experimental data in goat discs suggest that proteoglycan loss may contribute significantly more markedly to extracellular matrix reduction in disc degeneration than collagen [6]. This supports our notion that the observed age-related changes in our study population are mostly driven by proteoglycan content and less so by changes in collagen concentration.

\section{Anulus}
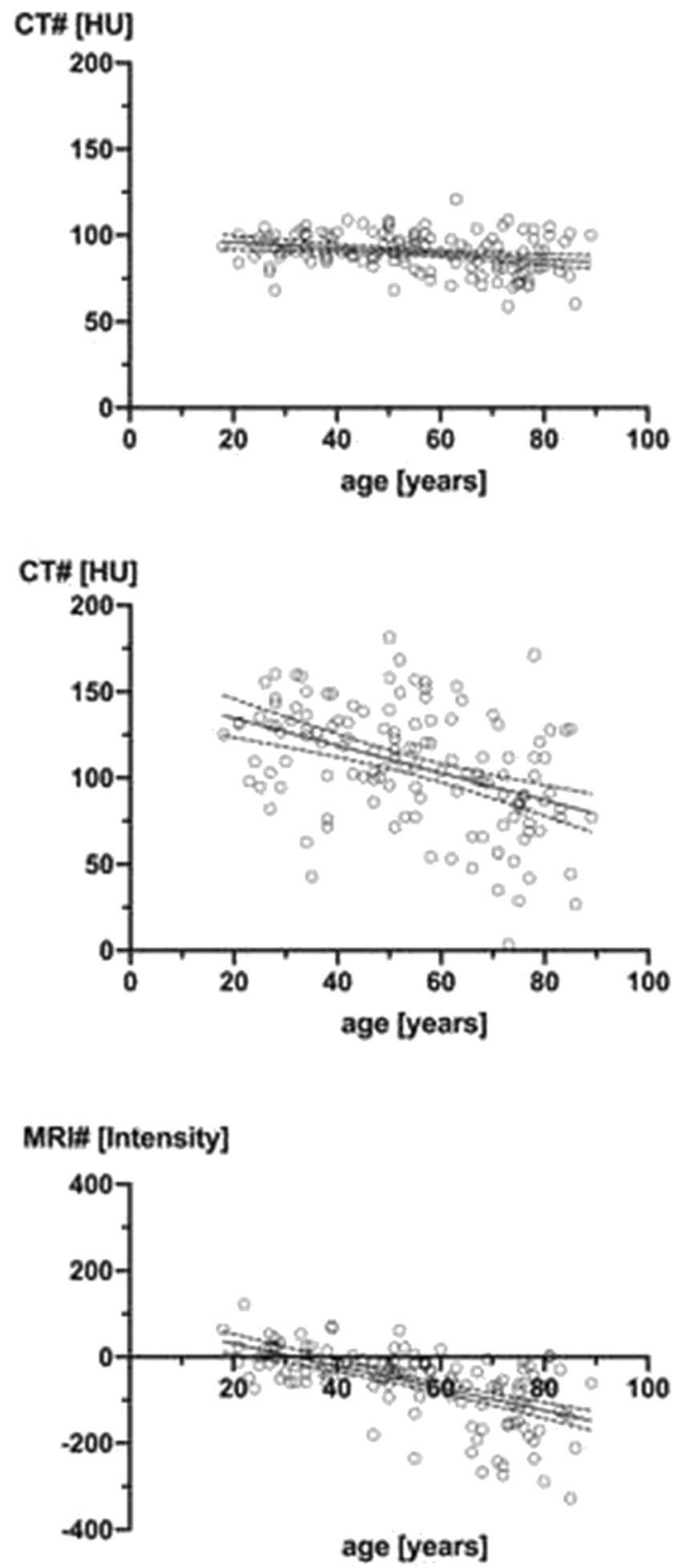

0.001; $r=-0.43$ ), suggesting an added value of these reconstructions. The MRI intensities of IVD compartments are inversely correlated with age. Relative intensities were calculated as IVD ROIs in the sagittal STIR sequence normalized by subtraction of ROIs in the paravertebral musculature. STIR intensity values significantly correlated with age at the AAF with $p=<0.0001 ; r=-0.60(95 \% \mathrm{CI}-0.70$ to -0.48$)$, and at the NP with $p=<0.0001 ; r=-0.74(95 \% \mathrm{CI}-0.81$ to -0.66$)$. STIR $=$ short-tau inversion recovery

When analyzing the age-related change in disc density, we controlled for the impact of different IVD levels on density. Both level Th12/L1 and L5/S1 were found to have significantly different densities compared with levels L1-5. We attribute this in part to the fact that only three IVDs of level Th12/L1 were analyzed. The higher density of L5/S1 may be related to the fact 
that this disc carries more weight than other levels. Moreover, in our patient population, most discs at this level were examined in very young patients, resulting in bias due to young age. Repeat correlation analysis of age-related density excluding levels Th12/L1 and L5/S1 proved a significant correlation between age and disc density in the AAF only.

Our data clearly show an age-dependent loss of IVD fluidity, as seen in the inverse correlation of STIR intensity of both AAF and NP in discs of different age. This is a known result of a concurrent loss of collagen/proteoglycans and water content predominantly in the NP $[21,22]$. Additional linear regression analysis revealed a significant reduction of density in the AAF per year of age, which was close to $1 \mathrm{HU}$ per year in the cMap. Recent data also confirm the role of DECT in the analysis of lumbar disc degeneration; however, the investigators used a different postprocessing technique that is nonspecific to disc material [18].

While others have analyzed IVDs on DECT VNCa maps, our approach is to use cMaps for IVD visualization. VNCa virtually subtracts calcium from the image, providing indirect measurements of the collagen content similar to conventional CT. cMaps, however, measure the target substances directly by their dual-energy absorption. We previously reported on a patient with spondylodiscitis in whom we demonstrated pronounced loss of signal in cMaps reflecting the extent of disc destruction [23]. We were also able to show that DECT can be sensitive to microstructural disc injuries in patients with vertebral compression fractures [17]. More recently, we were able to show the IVD morphology on cMaps with excellent correlation between DECT and MRI in disc herniation [24]. These results support the accuracy of IVD imaging in various spine pathologies. The current study lays the groundwork for future investigation into the potential of disc imaging with collagenand proteoglycan-sensitive DECT reconstruction. Also, follow-up imaging using DECT may play a role in the evaluation of microstructural IVD regeneration, while assessing new therapeutic targets [25]. In our data, no gender-related differences in density of the IVD were noted.

While we took care in planning and conducting our phantom experiments, some limitations have to be discussed. First, the preparation of collagen for the in vitro phantom setup yields a heterogeneous fluid, resulting in greater variance of the acquired data compared with chondroitin sulfate. Still, the authors consider these results relevant, as an impact of chondroitin sulfate on DECT cMap density was not reported previously. Second, the analysis of age-dependent changes in IVD microstructure stems from a retrospective study design, which limits the conclusions that can be drawn from these data. This retrospective analysis included CT scans acquired for various reasons (e.g., fracture, septic discitis, and axial spondyloarthritis); however, a certain inclusion bias might be present. Also, all intervertebral discs were morphologically assessed using both MRI and DECT images.

\section{Conclusion}

DECT imaging of IVDs is an emerging imaging option for patients with various spine pathologies. Our results reveal proteoglycans as a major contributor to disc density in DECT imaging besides collagen. Age-related changes in IVD microstructure can be depicted by DECT, and dedicated reconstructions (cMaps) may yield supplementary information compared to conventional CT images.

Supplementary Information The online version contains supplementary material available at https://doi.org/10.1007/s00330-021-08028-z.

Acknowledgements The authors wish to thank Bettina Herwig for correcting the manuscript.

Funding Open Access funding enabled and organized by Projekt DEAL. Prof. Bernd Hamm received funding from the Deutsche Forschungsgemeinschaft (372486779-SFB 1340.

The dept. of radiology received research grants from Canon medical systems.

\section{Declarations}

Guarantor The scientific guarantor of this publication is Julian Pohlan.

Conflict of interest The authors of this manuscript declare relationships with the following companies: Canon Medical Systems; i.e., Torsten Diekhoff held lectures for Canon medical systems.

The authors declare no other relationships with any companies whose products or services may be related to the subject matter of the article.

Statistics and biometry One of the authors (TD) has significant statistical expertise. No complex statistical methods requiring further support by biometry were necessary for this paper.

Informed consent Written informed consent was obtained from part of the subjects (patients) in this study - please compare study arm patients with osteoporotic vertebral fractures (Pumberger et al, 2020). Written informed consent was not required for the remainder of the human part of this study because of its retrospective design.

Ethical approval Institutional Review Board approval was obtained.

Study subjects or cohorts overlap Some study subjects have been previously reported; i.e., the patients with osteoporotic vertebral fractures were differently analyzed in another study published in this journal under DOI 10.1007/s00330-018-5963-4. Please compare with the following section in the manuscript: "Specifically, we included patients $[\ldots]$ with osteoporotic vertebral fractures $(n=68)[\ldots]$."

\section{Methodology}

- retrospective (with reference to the intervertebral disc analysis in patients); the experimental setup was prospective in nature

- observational and experimental

- performed at one institution

Open Access This article is licensed under a Creative Commons Attribution 4.0 International License, which permits use, sharing, adaptation, distribution and reproduction in any medium or format, as long as 
you give appropriate credit to the original author(s) and the source, provide a link to the Creative Commons licence, and indicate if changes were made. The images or other third party material in this article are included in the article's Creative Commons licence, unless indicated otherwise in a credit line to the material. If material is not included in the article's Creative Commons licence and your intended use is not permitted by statutory regulation or exceeds the permitted use, you will need to obtain permission directly from the copyright holder. To view a copy of this licence, visit http://creativecommons.org/licenses/by/4.0/.

\section{References}

1. Dowdell J, Erwin M, Choma T et al (2017) Intervertebral disk degeneration and repair. Neurosurgery. 80:S46-s54. https://doi. org/10.1093/neuros/nyw078

2. Farshad-Amacker NA, Hughes A, Herzog RJ, Seifert B, Farshad M (2017) The intervertebral disc, the endplates and the vertebral bone marrow as a unit in the process of degeneration. Eur Radiol 27: 2507-2520. https://doi.org/10.1007/s00330-016-4584-z

3. Raj PP (2008) Intervertebral disc: anatomy-physiology-pathophysiology-treatment. Pain Pract 8:18-44. https://doi.org/10.1111/j. 1533-2500.2007.00171.x

4. Lyons G, Eisenstein SM, Sweet MB (1981) Biochemical changes in intervertebral disc degeneration. Biochim Biophys Acta 673: 443-453. https://doi.org/10.1016/0304-4165(81)90476-1

5. Hu B, Xu C, Tian Y et al (2017) Inflammatory microRNA-194 and -515 attenuate the biosynthesis of chondroitin sulfate during human intervertebral disc degeneration. Oncotarget 8:49303-49317. https://doi.org/10.18632/oncotarget.17571

6. Emanuel KS, Mader KT, Peeters M et al (2018) Early changes in the extracellular matrix of the degenerating intervertebral disc, assessed by Fourier transform infrared imaging. Osteoarthr Cartil 26:1400-1408. https://doi.org/10.1016/j.joca.2018.06.003

7. Brinjikji W, Luetmer PH, Comstock B et al (2015) Systematic literature review of imaging features of spinal degeneration in asymptomatic populations. AJNR Am J Neuroradiol 36:811-816. https://doi.org/10.3174/ajnr.A4173

8. Frobin W, Brinckmann P, Kramer M, Hartwig E (2001) Height of lumbar discs measured from radiographs compared with degeneration and height classified from MR images. Eur Radiol 11:263269. https://doi.org/10.1007/s003300000556

9. Ling W, Regatte RR, Navon G, Jerschow A (2008) Assessment of glycosaminoglycan concentration $<$ em $>$ in vivo $<$ /em $>$ by chemical exchange-dependent saturation transfer (gagCEST). Proc Natl Acad Sci 105:2266-2270. https://doi.org/10.1073/pnas. 0707666105

10. Russo RJ, Costa HS, Silva PD et al (2017) Assessing the risks associated with MRI in patients with a pacemaker or defibrillator. N Engl J Med 376:755-764. https://doi.org/10.1056/ NEJMoa1603265

11. Shulman RM, Hunt B (2018) Cardiac implanted electronic devices and MRI safety in 2018-the state of play. Eur Radiol 28:40624065. https://doi.org/10.1007/s00330-018-5396-0

12. Manchikanti L, Soin A, Benyamin RM et al (2018) An update of the systematic appraisal of the accuracy and utility of discography in chronic spinal pain. Pain Physician 21:91-110
13. Wolfer LR, Derby R, Lee JE, Lee SH (2008) Systematic review of lumbar provocation discography in asymptomatic subjects with a meta-analysis of false-positive rates. Pain Physician 11:513-538

14. McCormick ZL, Lehman VT, Plastaras CT et al (2019) Lowpressure lumbar provocation discography according to spine intervention society/International Association for the Study of Pain standards does not cause acceleration of disc degeneration in patients with symptomatic low back pain: a 7-year matched cohort study. Spine (Phila Pa 1976) 44:E1161-E11E8

15. Manchikanti L, Benyamin RM, Singh V et al (2013) An update of the systematic appraisal of the accuracy and utility of lumbar discography in chronic low back pain. Pain Physician 16:Se55-Se95

16. Jeon JY, Lee SW, Jeong YM, Yu S (2019) The utility of dualenergy CT collagen material decomposition technique for the visualization of tendon grafts after knee ligament reconstruction. Eur J Radiol 116:225-230. https://doi.org/10.1016/j.ejrad.2019.03.012

17. Pumberger M, Fuchs M, Engelhard N et al (2019) Disk injury in patients with vertebral fractures - a prospective diagnostic accuracy study using dual-energy computed tomography. Eur Radiol 1-8

18. Shinohara Y, Sasaki F, Ohmura T et al (2020) Evaluation of lumbar intervertebral disc degeneration using dual energy CT virtual noncalcium imaging. Eur J Radiol 124:108817. https://doi.org/10. 1016/j.ejrad.2020.108817

19. Booz C, Nöske J, Martin SS et al (2019) Virtual noncalcium dualenergy CT: detection of lumbar disk herniation in comparison with standard gray-scale CT. Radiology. 290:446-455. https://doi.org/ 10.1148/radiol.2018181286

20. Martins DE, Medeiros VP, Wajchenberg M et al (2018) Changes in human intervertebral disc biochemical composition and bony end plates between middle and old age. PLoS One 13:e0203932. https:// doi.org/10.1371/journal.pone.0203932

21. Marinelli NL, Haughton VM, Muñoz A, Anderson PA (2009) T2 relaxation times of intervertebral disc tissue correlated with water content and proteoglycan content. Spine (Phila Pa 1976) 34:520 524. https://doi.org/10.1097/BRS.0b013e318195dd44

22. Li H, Yan JZ, Chen YJ, Kang WB, Huang JX (2017) Non-invasive quantification of age-related changes in the vertebral endplate in rats using in vivo DCE-MRI. J Orthop Surg Res 12:169. https:// doi.org/10.1186/s13018-017-0669-x

23. Pohlan J, Cink V, Schömig F, Pumberger M, Diekhoff T (2020) Pyogenic spondylodiscitis: dual-energy computed tomography reveals disc destruction in an 80-year-old patient. Eurorad. https://doi. org/10.35100/eurorad/case.16622

24. Schömig F, Pumberger M, Palmowski Y et al (2020) Vertebral disk morphology of the lumbar spine: a retrospective analysis of collagen-sensitive mapping using dual-energy computed tomography. Skeletal Radiol. https://doi.org/10.1007/s00256-020-03685-5

25. Sakai D, Nakai T, Hiraishi S et al (2018) Upregulation of glycosaminoglycan synthesis by neurotropin in nucleus pulposus cells via stimulation of chondroitin sulfate $\mathrm{N}$-acetylgalactosaminyltransferase 1: a new approach to attenuation of intervertebral disc degeneration. PLoS One 13:e0202640. https://doi.org/10.1371/journal.pone.0202640

Publisher's note Springer Nature remains neutral with regard to jurisdictional claims in published maps and institutional affiliations. 